\title{
Efeito benéfico da correção da acidose metabólica no estado nutricional de pacientes em hemodiálise
}

\author{
Beneficial effects of metabolic acidosis correction in \\ hemodialysis patients
}

\section{Autores}

Eduila Maria Couto Santos ${ }^{1}$

Marina de Moraes Vasconcelos Petribú ${ }^{1}$

Ana Paula Santana Gueiros $^{2}$

José Edevanilson de Barros Gueiros ${ }^{2}$

Poliana Coelho Cabral ${ }^{3}$

Florisbela de Arruda Câmara e Siqueira Campos $^{3}$

Suzana Meira de Oliveira $^{4}$

Emídio Cavalvanti de Albuquerque $^{5}$

'Universidade Federal de Pernambuco/Centro Acadêmico de Vitória de Santo Antão - Vitória de Santo Antão, PE, Brasil ${ }^{2}$ Hospital das Clínicas Universidade Federal de Pernambuco - Recife, PE, Brasil

${ }^{3}$ Universidade Federal de Pernambuco - Recife, PE, Brasil

${ }^{4}$ Universidade Federal de Pernambuco - Recife, PE,

Brasil

${ }^{5}$ Instituto Materno-Infantil

Professor Fernando Figueira

- Recife, PE, Brasil

Data de submissão: 08/06/2009 Data de aprovação: 11/09/2009

\section{Correspondência para:} Eduila Maria Couto Santos. Rua José Braz Moscow, 2075, apt. 401. Edf. Park Piedade

CEP: $54410-390$

Tel: 8787-7007

E-mail: eduila@hotmail.com

Declaramos a inexistência de conflitos de interesse.

\section{Resumo}

Objetivo: Avaliar o efeito da correção da acidose metabólica no estado nutricional de pacientes em hemodiálise. Métodos: Foram estudados, durante seis meses, 20 pacientes com acidose metabólica, definida pela média de três mensurações de bicarbonato sérico pré-diálise $<22 \mathrm{mEq} / \mathrm{L}$. Os pacientes dialisavam há, pelo menos, seis meses, utilizando bicarbonato de $35 \mathrm{mEq} / \mathrm{L}$ no dialisato. A correção da acidose metabólica foi feita mediante elevação do bicarbonato no dialisato para valores que não ultrapassaram $40 \mathrm{mEq} / \mathrm{L}$, objetivando um bicarbonato sérico entre $22-26 \mathrm{mEq} / \mathrm{L}$. Foram avaliados no início e no final do estudo: avaliação antropométrica, dietética, bioquímica e Avaliação Subjetiva Global (ASG). Resultados: A avaliação nutricional na fase inicial do estudo demonstrou índice de massa corporal normal $\left(24,23 \pm 3,83 \mathrm{~kg} / \mathrm{m}^{2}\right)$. A circunferência muscular do braço, a prega cutânea tricipital e a ASG classificaram homens e mulheres como desnutridos. Os consumos de calorias e proteínas foram $29,7 \pm 10,1$ $\mathrm{kcal} / \mathrm{kg} / \mathrm{dia}$ e 1,31 $\pm 0,35 \mathrm{~g} / \mathrm{kg} / \mathrm{dia}$, respectivamente. A avaliação bioquímica observou albumina sérica normal e colesterol reduzido. Após correção, bicarbonato sérico e $\mathrm{pH}$ aumentaram de $18,2 \pm 1,64$ para $22 \pm 1,70$ $(\mathrm{p}<0,001)$, e de 7,32 $\pm 0,45$ para 7,37 \pm $0,41(\mathrm{p}<0,001)$, respectivamente. Houve melhora da ASG $(21,7 \pm 6,4$ versus $16,8 \pm$ $6,6, \mathrm{p}<0,001)$ e aumento na ingestão calórica $(1.892,61 \pm 454,30$ versus $2.110,30 \pm$ $869,24, \mathrm{p}<0,05)$. Conclusão: A suplementação de bicarbonato na solução de hemodiálise foi método efetivo para a correção da acidose metabólica, determinando aumento da ingestão calórica e melhora nos escores da ASG.

Palavras-chave: hemodiálise, acidose metabólica, estado nutricional.

[J Bras Nefrol 2009;31(4):244-251]@Elsevier Editora Ltda.

\section{Abstract}

Objective: To assess the nutritional effect of correcting metabolic acidosis in hemodialysis patients. Methods: Twenty patients with metabolic acidosis, defined as mean serum bicarbonate $<22 \mathrm{mEq} / \mathrm{L}$ before dialysis, were studied for six months. They had been on dialysis for at least six months, with $35 \mathrm{mEq} / \mathrm{L}$ of bicarbonate in the dialysate. Metabolic acidosis was corrected through elevation in dialysate bicarbonate to values not exceeding 40 $\mathrm{mEq} / \mathrm{L}$, aiming at bicarbonate serum levels between 22 and $26 \mathrm{mEq} / \mathrm{L}$. Biochemical, anthropometric, and dietary assessments were performed at the beginning and end of the study, as was Global Subjective Assessment (GSA). Results: The nutritional assessment in the initial phase of the study showed normal body mass index (24.23 \pm $\left.3.83 \mathrm{~kg} / \mathrm{m}^{2}\right)$. However, according to arm muscle circumference, triceps skinfold, and GSA, men and women were classified as undernourished. Calorie and protein intakes were $29.7 \pm 10.1 \mathrm{kcal} / \mathrm{kg} /$ day and $1.31 \pm 0.35 \mathrm{~g} / \mathrm{kg} /$ day, respectively. The biochemical evaluation showed normal serum albumin and low cholesterol. After correction, serum bicarbonate and $\mathrm{pH}$ increased from $18.2 \pm 1.64$ to $22 \pm 1.70$ $(\mathrm{p}<0.001)$ and from $7.32 \pm 0.45$ to 7.37 $\pm 0.41(\mathrm{p}<0.001)$, respectively. GSA improved $(21.7 \pm 6.4$ versus $16.8 \pm 6.6, \mathrm{p}<$ $0.001)$ and calorie intake increased (1892 \pm 454.30 versus $2110.30 \pm 869.24, \mathrm{p}<$ 0.05). Conclusions: Bicarbonate supplementation in hemodialysis solution was effective for correcting metabolic acidosis, determining an increase in calorie intake and improvement in GSA scores.

Keywords: hemodialysis, metabolic acidosis, nutritional status. 


\section{INTRODUÇÃO}

Desnutrição energético-proteica (DEP), caracterizada por redução da massa muscular, anormalidades no metabolismo de aminoácidos e reduzidas concentrações de proteínas séricas, ${ }^{1}$ constitui complicação prevalente da insuficiência renal crônica (IRC), contribuindo de forma importante para a morbidade e a mortalidade dos pacientes renais crônicos, principalmente aqueles em diálise. ${ }^{2}$

Vários são os fatores que predispõem à DEP em pacientes em hemodiálise: o procedimento dialítico resulta em perda de nutrientes, aumento do catabolismo $^{3}$ e diminuição da síntese de proteínas; ${ }^{4}$ os sintomas de uremia, incluindo anorexia, náuseas e vômitos, reduzem a ingestão calórica e proteica; ${ }^{5}$ a resistência à insulina resulta em aceleração da atrofia muscular ${ }^{6}$ e acidose metabólica, por ser catabólica e antianabólica. $^{7}$

Acidose metabólica é frequente em pacientes com IRC, principalmente naqueles em estágios mais avançados. ${ }^{8}$ Um dos objetivos do tratamento dialítico é corrigir o desequilíbrio ácido-base. No entanto, a acidose metabólica é mantida em um número considerável de pacientes submetidos à hemodiálise, a despeito de um bom índice de adequação dialítica. ${ }^{1,9,10}$

A acidose metabólica desenvolve papel importante na patogênese da DEP em pacientes renais crônicos, por inibir a síntese proteica em diferentes tecidos ${ }^{10,11} \mathrm{e}$ estimular a degradação proteica e a oxidação de aminoácidos de cadeia ramificada, resultando em balanço nitrogenado negativo. ${ }^{12}$ Estes efeitos são dependentes de glicocorticoide e mediados pela protease caspase- $3^{13}$ do sistema ubiquitina-proteasome, dependente de ATP e da enzima cetoácido-desidrogenase. ${ }^{14}$

Vários estudos clínicos prospectivos observaram o efeito benéfico da correção da acidose metabólica em pacientes renais crônicos submetidos à diálise. Alguns autores demonstraram redução da proteólise muscular e da oxidação de aminoácidos de cadeia ramificada, ${ }^{1,15}$ enquanto outros verificaram elevação da concentração da albumina sérica, ${ }^{16}$ melhora significativa dos parâmetros antropométricos, como pregas cutâneas e circunferência muscular do braço, ${ }^{17} \mathrm{e}$ redução da morbidade, com menor tempo de internação. ${ }^{18}$ No entanto, outros pesquisadores não demonstraram benefícios nutricionais da correção da acidose metabólica. ${ }^{19,20}$

Considerando que a acidose metabólica é um importante fator na etiologia da desnutrição em pacientes com IRC, e que a normalização do desequilíbrio ácido-base reverte os seus efeitos deletérios sobre o estado nutricional, o objetivo deste estudo foi avaliar o efeito da correção da acidose metabólica no estado nutricional de uma população em hemodiálise em um centro de tratamento de Pernambuco/Brasil.

\section{Metodologia}

\section{Casuística}

Este estudo foi previamente aprovado pelo Comitê de Ética, para estudos em humanos, do Centro de Ciências da Saúde (CEP/ CCS/ UFPE), sob o registro $n^{\circ}$ 113/06, de acordo com a resolução no $196 / 96$ do Conselho Nacional de Saúde.

Este foi um estudo prospectivo, realizado em um centro de hemodiálise de Pernambuco, no período de novembro de 2006 a julho de 2007 , no qual 47 pacientes encontravam-se regularmente matriculados. Foram selecionados 30 pacientes, de acordo com os seguintes critérios de elegibilidade: idade maior ou igual a 18 anos, tempo de hemodiálise superior a seis meses, acesso vascular por fístula arteriovenosa e ausência de outras patologias crônicas ou consumptivas (foram excluídos pacientes portadores do vírus HIV, hepatopatias e neoplasias malignas).

Todos os pacientes dialisavam em máquinas modelo Tina, da Baxter ${ }^{\circledR}$, com membrana de polissulfona, capilar F8 (Fresenius ${ }^{\circledR}$ ), fluxo de sangue entre 350 e $400 \mathrm{~mL} / \mathrm{min}$, fluxo de dialisato de $500 \mathrm{~mL} / \mathrm{min} \mathrm{e}$ utilizavam soluções de diálise contendo $3,5 \mathrm{mEq} / \mathrm{L}$ de cálcio e $35 \mathrm{mEq} / \mathrm{L}$ de bicarbonato. A duração da diálise era de quatro horas, com frequência de três vezes por semana. Dezoito pacientes faziam uso de quelante de fósforo, sendo que dez utilizavam o carbonato de cálcio e oito, sevelamer.

\section{Protocolo do estudo}

Os pacientes foram submetidos a três mensurações do bicarbonato sérico, realizadas em meses consecutivos. Acidose metabólica foi definida quando a média aritmética destas medidas foi inferior a $22 \mathrm{mEq} / \mathrm{L} .{ }^{21} \mathrm{~A}$ amostra de sangue venoso foi retirada no início da diálise, após o maior intervalo interdialítico, e analisada dentro de 30 minutos para evitar diminuição da concentração de bicarbonato em razão do retardo na mensuração.

Os pacientes foram acompanhados por seis meses, o que constituiu o período de intervenção. Durante este período, a correção da acidose metabólica foi realizada mediante programação digital na máquina de hemodiálise, elevando a concentração de bicarbonato no dialisato para a faixa entre 36 e $40 \mathrm{mEq} / \mathrm{L}$. Objetivou-se alcançar um bicarbonato sérico entre 22 
e $26 \mathrm{mEq} / \mathrm{L}$ e, para tal, gasometria venosa era obtida mensalmente para monitorização desta intervenção. Durante todo o período estudado, os demais parâmetros da prescrição dialítica não foram alterados.

Foram avaliados os seguintes parâmetros clínicos e demográficos: idade, sexo, etiologia da doença renal, função renal residual (FRR) e tempo de diálise. A presença de FRR foi definida por diurese $>200 \mathrm{~mL} / 24 \mathrm{~h}$

A avaliação do estado nutricional foi realizada no início e no término do estudo, mediante a determinação de indicadores bioquímicos, antropométricos, dietéticos e da avaliação subjetiva global (ASG).

Os parâmetros laboratoriais foram determinados em amostra pré-dialítica de sangue venoso: $\mathrm{pH}$ sanguíneo (7,31-7,41; método: gasometria venosa GEM 3000); concentração de bicarbonato sérico (22-26 mEq/L; método: gasometria venosa GEM 3000); ureia (17 - $43 \mathrm{mg} / \mathrm{dL}$; método: urease - gldh diasys); creatinina $(0,6-1,4 \mathrm{mg} / \mathrm{dL}$; método: architect abbott); albumina $(3,5-5,2 \mathrm{~g} / \mathrm{dL}$; método: verde de bromocresol diasys); colesterol total (150-99 mg/ dL; método: cho - pap diasys). No início do estudo, amostra pós-dialítica de sangue foi retirada para determinação da ureia plasmática e posterior cálculo do $\mathrm{Kt} / \mathrm{V}$, considerando-se adequados os valores superiores a $1,2 .{ }^{22}$

As mensurações antropométricas, realizadas após a sessão de hemodiálise, foram: Índice de Massa Corporal (IMC), calculado pelo peso seco e avaliado de acordo com a classificação da WHO, $1995 ;{ }^{23}$ prega cutânea tricipital (PCT) e circunferência muscular do braço (CMB), classificadas de acordo com o padrão de referência de Frisancho ${ }^{24}$ e medidas no braço sem fístula.

A ASG foi realizada por única nutricionista, conforme descrito por Kalantar-Zadeh et al. ${ }^{25}$ Segundo estes autores, cada item deve ser pontuado de acordo com a intensidade da alteração encontrada, variando de normal ( 1 ponto) a muito grave (5 pontos). Um escore de desnutrição é obtido mediante o somatório dos pontos, sendo que os pacientes eutróficos têm valores igual a 7 e os gravemente desnutridos, próximos a 35 .

Para análise do consumo alimentar, utilizou-se o registro alimentar de três dias, incluindo um dia do final de semana, um dia do período interdialítico e um dia da diálise, ${ }^{26}$ sendo avaliadas as variações intra e interpessoal. Todos os pacientes foram orientados por nutricionista para a realização do diário alimentar. As recomendações nutricionais para pacientes em tratamento dialítico foram estipuladas de acordo com as necessidades nutricionais individuais, conforme descritos por Martins \& Riella,2001 - 30 a 35 kcal/ $\mathrm{kg} / \mathrm{dia}$ e 1,2 a $1,4 \mathrm{~g}$ de proteína $/ \mathrm{kg} / \mathrm{dia} .{ }^{27}$

\section{Processamento e anÁlise dos dados:}

A construção do banco de dados foi realizada no programa Epi Info, versão $6.04 .{ }^{28} \mathrm{O}$ programa estatístico SPSS foi utilizado para avaliar o comportamento das variáveis quantitativas segundo o critério de normalidade da distribuição e nas subsequentes análises estatísticas. $^{29}$

A comparação entre as médias (dados paramétricos) foi realizada pelo teste t de Student pareado. Foi adotado o nível de significância de 5\% para rejeição da hipótese de nulidade.

A análise da composição da dieta foi realizada por meio do software de apoio a Nutrição da Escola Paulista de Medicina (1993). ${ }^{30}$ A tabela base deste programa é a do Departamento de Agricultura dos Estados Unidos, ano 1976-1986. No entanto, em virtude da ocorrência de inúmeros produtos de consumo regional, alguns alimentos foram analisados pela Tabela de Franco/Brasil (1997). ${ }^{31}$ Como o registro foi feito em medidas caseiras, houve a necessidade de conversão destas em gramas, utilizando-se como padrão de referência a Tabela de Pinheiro et al., 1994. ${ }^{32}$

\section{Resultados}

Dos 30 pacientes selecionados, 27 apresentavam acidose metabólica $(90 \%)$ e, destes, 20 concluíram o período de observação e compuseram a amostra estudada. As perdas na amostra ocorreram por óbito (um paciente), internação hospitalar (dois pacientes), transferência (um paciente) e mudança na modalidade dialítica (três pacientes).

Os pacientes estavam em diálise por um período de 40,2 $\pm 26,9$ meses, com boa eficiência dialítica $(\mathrm{Kt} / \mathrm{V}=1,68 \pm 0,24)$. A idade média era de 51,7 \pm 12,9 anos. Dez pacientes eram do sexo masculino e 11 ainda apresentavam FRR. Com relação à etiologia da doença renal, $35 \%$ dos pacientes não apresentavam diagnóstico confirmado; nefropatia diabética e nefroesclerose hipertensiva representaram, cada uma, $15 \%$ da amostra.

Os resultados descritos a seguir se referem à avaliação nutricional mensurada na fase inicial do estudo.

A Tabela 1 mostra os dados referentes à avaliação antropométrica. A média do IMC esteve dentro da faixa de normalidade. A análise da CMB definiu desnutrição leve e eutrofia para os sexos masculino e feminino, respectivamente. Já a PCT classificou como desnutridos ambos, homens e mulheres. 


\begin{tabular}{|ccl|}
\hline Tabela 1 & $\begin{array}{l}\text { AVALIAÇÃO ANTROPOMÉTRICA DE PACIENTES COM ACIDOSE METABÓLICA MANTIDOS EM } \\
\text { HEMODIÁLISE }(\mathrm{N}=20)\end{array}$ & Diagnóstico \\
\hline Variável & Média $\pm \mathrm{DP}$ & Eutrofia \\
\hline $\mathrm{IMC}\left(\mathrm{kg} / \mathrm{m}^{2}\right)$ & $24,23 \pm 3,83$ & \\
\hline $\mathrm{CMB}(\mathrm{cm})$ & & Desnutrição leve \\
\hline Masculino & $24,26 \pm 2,23$ & Eutrofia \\
\hline Feminino & $23,63 \pm 2,64$ & \\
\hline PCT $(\mathrm{mm})$ & & Desnutrição leve \\
\hline Masculino & $9,93 \pm 5,59$ & Desnutrição leve \\
\hline Feminino & $27,06 \pm 5,68$ & \\
\hline
\end{tabular}

IMC = Índice de Massa Corporal

$\mathrm{CMB}=$ Circunferência Muscular do Braço

$\mathrm{PCT}=$ Prega Cutânea Tricipital

No Gráfico 1, pode-se observar a classificação do estado nutricional de acordo com a ASG, a qual revelou déficit nutricional em todos os pacientes.

$\mathrm{Na}$ Tabela 2, observamos os resultados dos parâmetros laboratoriais. A média do bicarbonato sérico foi de 18,2 $\pm 1,64 \mathrm{mEq} / \mathrm{L}$ e o $\mathrm{pH}, 7,33 \pm 0,04$. Observou-se albumina sérica dentro dos parâmetros de normalidade $(3,77 \pm 0,33 \mathrm{~g} / \mathrm{dL})$.

Com relação à avaliação dietética, o consumo de calorias foi de 1.892,6 $\pm 454,29 \mathrm{kcal}$ e o de proteínas, de $83,7 \pm 18,6$ g. Em média, os pacientes ingeriram 29,7 \pm $10,1 \mathrm{kcal} / \mathrm{kg} /$ dia e $1,31 \pm 0,35 \mathrm{~g}$ de proteína $/ \mathrm{kg} / \mathrm{dia}$.

$\mathrm{O}$ efeito da correção da acidose metabólica sobre os parâmetros laboratoriais e nutricionais estão apresentados na Tabela 3. Observa-se elevação do $\mathrm{pH}$ sanguíneo $(7,3 \pm 0,05$ versus $7,4 \pm 0,04 ; p=0,000)$ e do bicarbonato $(18,2 \pm 1,64$ versus $21,0 \pm 1,72 ; \mathrm{p}=0,000)$. Os parâmetros antropométricos mantiveram-se estáveis em todo o período estudado. Houve aumento da ingestão calórica $(1.892,60 \pm 454,29$ versus $2.110,00$ $\pm 170,26 ; \mathrm{p}=0,026)$ e melhora dos escores da ASG $(21,70 \pm 6,40$ versus $16,80 \pm 6,60 ; p=0,000)$.
Gráfico 1 - Estado nutricional segundo a Avaliação Subjetiva Global em pacientes com acidose metabólica mantidos em hemodiálise $(\mathrm{n}=20)$.

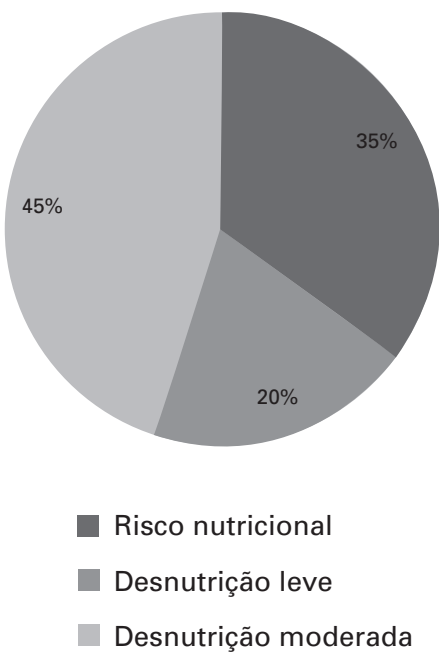

Tabela 2 PARÂMETROS LABORATORIAIS DE PACIENTES COM ACIDOSE METABÓLICA MANTIDOS EM HEMODIÁLISE $(N=20)$

\begin{tabular}{|ll|}
\hline Variável & Média $\pm \mathrm{DP}$ \\
\hline $\mathrm{pH}$ & $7,3 \pm 0,05$ \\
\hline $\mathrm{pCO}_{2}(\mathrm{mmHg})$ & $34,7 \pm 4,11$ \\
\hline Bicarbonato $(\mathrm{mmol} / \mathrm{L})$ & $18,2 \pm 1,64$ \\
\hline Ureia $(\mathrm{mg} / \mathrm{dL})$ & $166,2 \pm 43,96$ \\
\hline Creatinina $(\mathrm{mg} / \mathrm{dL})$ & $10,0 \pm 2,68$ \\
\hline Albumina $(\mathrm{mg} / \mathrm{dL})$ & $3,8 \pm 0,27$ \\
\hline Colesterol total $(\mathrm{mg} / \mathrm{dL})$ & $158,3 \pm 43,43$ \\
\hline
\end{tabular}




\begin{tabular}{|c|c|c|c|c|}
\hline \multicolumn{4}{|c|}{$\begin{array}{l}\text { PARÂMETROS LABORATORIAIS E NUTRICIONAIS DE PACIENTES MANTIDOS EM } \\
\text { HEMODIÁLISE PRÉ E PÓS-CORREÇÃO DA ACIDOSE METABÓLICA }(\mathrm{N}=20)\end{array}$} & \multirow{3}{*}{ p-valor * } \\
\hline \multirow{2}{*}{\multicolumn{2}{|c|}{ Variáveis }} & \multicolumn{2}{|c|}{ Momento } & \\
\hline & & Tempo 0 & Tempo 6 & \\
\hline \multicolumn{5}{|c|}{ Gasometria Venosa } \\
\hline $\mathrm{pH}$ & & $7,3 \pm 0,05$ & $7,4 \pm 0,04$ & $0,000 a$ \\
\hline $\mathrm{pCO}$ & $\mathrm{mHg})$ & $34,7 \pm 4,11$ & $37,6 \pm 3,72$ & $0,011 a$ \\
\hline Bicar & רato (mmol/L) & $18,2 \pm 1,64$ & $21,9 \pm 1,72$ & $0,000 a$ \\
\hline \multicolumn{5}{|c|}{ Avaliação Antropométrica } \\
\hline IMC & $\left(\mathrm{m}^{2}\right)$ & $24,6 \pm 3,58$ & $25,0 \pm 3,71$ & 0,108 \\
\hline PCT & & $18,6 \pm 6,27$ & $19,2 \pm 7,06$ & 0,323 \\
\hline $\mathrm{CMB}$ & & $24,2 \pm 1,99$ & $24,5 \pm 2,22$ & 0,261 \\
\hline \multicolumn{5}{|c|}{ Avaliação Subjetiva } \\
\hline ASG & & $21,7 \pm 6,4$ & $16,8 \pm 6,6$ & $0,000 a$ \\
\hline \multicolumn{5}{|c|}{ Avaliação Bioquímica } \\
\hline Ureia & g/dL) & $166,2 \pm 43,96$ & $164,4 \pm 40,58$ & 0,864 \\
\hline Crea & רa (mg/dL) & $10,0 \pm 2,68$ & $10,0 \pm 2,46$ & 0,939 \\
\hline Albu & $\mathrm{a}(\mathrm{mg} / \mathrm{dL})$ & $3,8 \pm 0,27$ & $3,8 \pm, 32$ & 0,930 \\
\hline Cole & ol total (mg/dL) & $158,3 \pm 43,43$ & $162,7 \pm 25,83$ & 0,744 \\
\hline \multicolumn{5}{|c|}{ Avaliação Dietética } \\
\hline Inge & calórica & $1892,6 \pm 454,29$ & $2110,0 \pm 170,26$ & 0,026a \\
\hline Inge & proteica & $83,7 \pm 18,62$ & $89,2 \pm 17,28$ & 0,185 \\
\hline
\end{tabular}

(*) Teste $t$ Student Pareado (a) $p<0,05$

IMC = Índice de Massa Corporal

$\mathrm{CMB}=$ Circunferência Muscular do Braço

PCT $=$ Prega Cutânea Tricipital

ASG = Avaliação Subjetiva Global

\section{Dıscussão}

A desnutrição é frequente em pacientes com IRC e está associada ao risco elevado de morbidade e mortalidade, principalmente em pacientes mantidos em diálise. ${ }^{2}$ Dentre os fatores associados à DEP de pacientes renais crônicos, a acidose metabólica desenvolve um importante papel, ${ }^{15}$ mediante mecanismos complexos de ativação da degradação proteica, oxidação de aminoácidos essenciais ${ }^{13}$ e inibição da síntese proteica, ${ }^{11}$ principalmente em estágios mais avançados da doença renal.

A terapia dialítica é responsável pelo clareamento das toxinas urêmicas e pelo controle do balanço hidroeletrolítico e ácido-base. ${ }^{33}$ Entretanto, vários são os trabalhos que demonstram que um número significativo de pacientes em diálise permanece acidótico, a despeito de bons resultados de Kt/V. ${ }^{9}{ }^{10}$ De fato, nosso trabalho encontrou uma alta prevalência de acidose metabólica $(90 \%)$ em pacientes hemodialisados com adequado $\mathrm{Kt} / \mathrm{V}$.

A solução tampão utilizada no dialisato é um dos fatores que influencia na habilidade da hemodiálise em corrigir o desequilíbrio ácido-base, ${ }^{34}$ no entanto, esta geralmente é arbitrária e varia de 32 a $35 \mathrm{mEq} / \mathrm{L} .^{34}$ Neste estudo, pôde-se observar que, após seis meses, a elevação de bicarbonato no dialisato promoveu aumento significante do $\mathrm{pH}$ e da concentração sérica de bicarbonato, de modo que $85 \%$ dos pacientes alcançaram nível sérico de bicarbonato pré-diálise entre 22 e $26 \mathrm{mEq} / \mathrm{L}$.

A avaliação do estado nutricional nos pacientes em hemodiálise é complexa, uma vez que as variações de água corporal podem influenciar nos índices antropométricos, a presença da fístula arteriovenosa dificulta a correta mensuração das medidas do braço e os padrões de referência não são específicos para esta população. Em função disso, a recomendação é a utilização de métodos múltiplos, aplicados simultaneamente. ${ }^{35}$ Neste contexto, a utilização da ASG modificada para pacientes em diálise tem-se mostrado um parâmetro mais válido na avaliação nutricional destes pacientes, por não sofrer as influências supracitadas. $^{36}$ 
A ASG é recomendada a pacientes mantidos em diálise, considerada válida e fortemente associada à mortalidade. ${ }^{36}$ Segundo o DOPPS, 2002, em um estudo prospectivo que avaliou risco de mortalidade em pacientes hemodialisados, aqueles com desnutrição severa, de acordo com o índice de ASG, apresentavam risco $33 \%$ maior de mortalidade do que os eutróficos. ${ }^{37}$ Em nosso estudo, evidenciamos um alto percentual de desnutrição segundo este parâmetro, sendo $45 \%$ considerados desnutridos moderados. Após o período de intervenção, melhora significativa do índice de ASG pôde ser observada, mostrando que $50 \%$ dos pacientes se mantiveram com desnutrição, mas apenas $10 \%$ foram classificados com grau moderado. À semelhança dos nossos resultados, Szeto et al., 2003, também relataram melhora no escore da ASG em pacientes em diálise peritoneal suplementados com bicarbonato de sódio oral. ${ }^{18}$ Entretanto, Blair et al., 2003, não observaram alteração no índice de ASG após correção da acidose metabólica por meio de metodologia semelhante a este estudo. ${ }^{38} \mathrm{~A}$ subjetividade deste indicador nutricional parece ser responsável pelos resultados variáveis, uma vez que a experiência e a habilidade do examinador influenciam o diagnóstico. ${ }^{2}$

Ao se avaliar os parâmetros antropométricos, verificou-se que os pacientes foram classificados como eutróficos, de acordo com o IMC. Quando os índices utilizados foram as medidas do braço, houve diferença entre os sexos, evidenciando-se desnutrição apenas no sexo masculino. Já quando avaliamos a PCT, ambos os sexos se mostraram desnutridos. Dados similares foram observados por Valenzuela et al., 2003, ao estudar pacientes renais crônicos submetidos à hemodiálise. Esses autores encontraram média do IMC dentro do limite da normalidade. No entanto, $45 \%$ dos seus pacientes apresentavam desnutrição identificada por PCT e/ou CMB $\leq$ percentil 5 do padrão de referência da população dos Estados Unidos. ${ }^{39} \mathrm{O}$ cálculo do IMC pode ser influenciado pela hiper-hidratação dos pacientes, a qual aumenta o peso corporal, mas tem pouca repercussão nas medidas da PCT e CMB. ${ }^{39} \mathrm{Na}$ tentativa de minimizar o erro na interpretação, as medidas antropométricas foram avaliadas após a sessão de hemodiálise do meio da semana, e o IMC foi calculado a partir do peso seco. Embora a melhor avaliação do volume de água corporal seja por meio da bioimpedância, ${ }^{40}$ neste estudo, infelizmente, não foi possível realizar tal procedimento, o que poderia ter enriquecido a análise dos nossos resultados.

Após a correção da acidose metabólica, não observamos modificações nos índices antropométricos.
Outros autores também não foram capazes de demonstrar alteração da composição corporal após seis meses de intervenção com a suplementação de bicarbonato. ${ }^{41}$ Por outro lado, Seyffart et al. mostraram um aumento significante do IMC em pacientes submetidos à hemodiálise quando a dose de bicarbonato no dialisato foi aumentada durante um período de 12 a 19 meses. $^{42}$ De forma semelhante, Williams et al., 1997, em um estudo duplo-cego conduzido por seis meses, encontraram aumento da PCT no grupo de pacientes com maiores concentrações de bicarbonato no dialisato. ${ }^{20}$ Assim, o impacto da correção da acidose metabólica sobre os parâmetros antropométricos não é concordante na literatura e, neste estudo, o tempo de intervenção pode ter sido insuficiente para determinar alguma mudança, uma vez que, tendo ocorrido aumento da ingestão calórica, era de se esperar um ganho de massa corpórea.

A albumina sérica, apesar das suas limitações, tem sido amplamente utilizada na avaliação nutricional do paciente renal crônico. ${ }^{26} \mathrm{Na}$ presença de inflamação aguda ou crônica, a síntese da albumina é prejudicada, limitando sua utilização como marcador do estado nutricional, mormente em pacientes dialíticos. ${ }^{36}$ Entretanto, a hipoalbuminemia é considerada preditor forte e independente de mortalidade em diálise. ${ }^{36}$ Blair et al., 2003, observaram que a elevação da concentração de bicarbonato no dialisato não resultou em aumento da albumina sérica. ${ }^{38}$ Por outro lado, Verove et al., 2002, avaliando o efeito da correção da acidose metabólica no estado nutricional de idosos com IRC, evidenciaram aumento dos níveis de albumina e pré-albumina após seis meses de suplementação de bicarbonato de sódio oral. ${ }^{43}$ Já Movilli et al., 1998, ao aumentar a concentração do bicarbonato no dialisato, demonstraram aumento da albumina sérica, mesmo em pacientes que já apresentavam níveis de albumina normais $(3,49 \pm 2,1 \mathrm{~g} / \mathrm{dL}$ versus $3,79 \pm 2,9$, $\mathrm{p}<0,01) .{ }^{16} \mathrm{~A}$ despeito do diagnóstico de desnutrição, os pacientes apresentavam níveis séricos normais de albumina, os quais se mantiveram após a correção da acidose metabólica. Pode-se sugerir que a manutenção $\mathrm{da}$ adequada ingestão proteica foi fator contribuinte para o resultado encontrado.

A baixa concentração plasmática de colesterol ( $<150 \mathrm{mg} / \mathrm{dL}$ ) é um fator preditivo de mortalidade, uma vez que se associa tanto a déficit nutricional quanto à inflamação. ${ }^{36}$ Pacientes em diálise que apresentam colesterol sérico entre 150 e $180 \mathrm{mg} / \mathrm{dL}$ devem ser avaliados para possíveis déficits nutricionais e para outras condições comórbidas. ${ }^{2}$ Segundo Kuhlmann et al., 2007, a concentração sérica de colesterol reflete 
ingestão calórica, não sendo afetada pela ingestão proteica. ${ }^{2}$ Esta pesquisa demonstrou que, após a correção da acidose metabólica, houve um aumento significante da ingestão calórica, ainda que ela tenha permanecido abaixo da recomendada. Pacientes com idade menor ou igual a 60 anos, característica desta amostra, deveriam ingerir cerca de $35 \mathrm{kcal} / \mathrm{kg} / \mathrm{dia} .^{27}$ Assim, o nível de colesterol encontrado pode refletir a baixa ingestão calórica observada nesta casuística. Apesar de não ter sido objetivo deste estudo, a avaliação de marcadores inflamatórios, os critérios de seleção dos pacientes e o fato deles apresentarem albumina sérica normal sugerem que a inflamação pode não ter sido fortemente presente nesta população.

O nível de creatinina sérica é proporcional à ingestão proteica e à massa muscular. ${ }^{2}$ Assim, é recomendado que pacientes com creatinina sérica pré-diálise inferior a $10 \mathrm{mg} / \mathrm{dL}$ devem ser avaliados para possível déficit proteico. ${ }^{2}$ Nesta pesquisa, os pacientes apresentavam adequados níveis de creatinina, antes e depois da correção da acidose metabólica, o que provavelmente pode ser explicado pela manutenção da ingestão proteica durante o período de observação, além do fato de a amostra estudada ser constituída de adultos jovens.

A ingestão deficiente de energia e proteínas tem sido colocada como uma das principais causas de desnutrição no tratamento dialítico. ${ }^{27}$ Estes resultados demonstraram que a ingestão calórica se mostrou inferior à recomendada para pacientes em diálise, enquanto o consumo proteico esteve adequado. Após a correção da acidose metabólica, os pacientes apresentaram um aumento significativo do consumo energético, provavelmente secundário à melhora do apetite, fato referido durante a ASG. Outros autores também mostraram que a correção da acidose metabólica apresentou um efeito positivo sobre o apetite, resultando em um aumento da ingestão dietética. ${ }^{18,44}$ Evidências na literatura têm demonstrado que um pobre apetite é fator de risco para hospitalização e, provavelmente, a melhora do apetite diminui o risco de mortalidade em pacientes hemodialisados. ${ }^{45}$ É importante ressaltar que o registro alimentar de três dias, realizado neste estudo, fornece uma estimativa confiável da ingestão dietética e é validado por diretrizes, como o K/DOQI, $2000 .^{26}$

Em conclusão, a suplementação de bicarbonato na solução de hemodiálise foi método efetivo para a correção da acidose, influenciando positivamente o estado nutricional, uma vez que se associou ao aumento da ingestão calórica e à melhora dos escores da ASG. Deste modo, a correção da acidose metabólica individualizada deve ser objetivo na conduta de pacientes mantidos em hemodiálise, a fim de minimizar os efeitos deletérios no estado nutricional e favorecer o prognóstico desta população a longo prazo.

\section{Agradecimentos}

À Fundação de Amparo à Ciência e Tecnologia do Estado de Pernambuco - FACEPE.

Ao Serviço de Nefrologia do Hospital das Clínicas Universidade Federal de Pernambuco.

\section{RefeÊnCIAS}

1. Kooman JP, Deutz NEP, Zijilmans P et al. The influence of bicarbonate supplementation on plasma levels of branched-chain amino acids in haemodialysis patients with metabolic acidosis. Nephrol Dial Transplant 1997; 12:2397-401.

2. Kuhlmann MK, Kribben A, Wittwer M, Horl WH. OPTA. Malnutrition in chronic renal failure. Nephrol Dial Transplant 2007; 22[Suppl. 3]:iii13-9.

3. Guiterrez A, Alvestrand A, Wahren J, Bergstrom J. The effect of in vivo contact between blood and dialysis membranes on protein catabolism in humans. Kidney Int 1990; 38:487-94.

4. Lim VS, Bier DM, Flanigan MJ, Sum-Ping ST. The effect of hemodialysis on protein metabolism. J Clin Invest 1993; 91:2429-36.

5. Wolfson M. Management of Protein and Energy Intake in Dialysis Patients. J Am Soc Nephrol 1999; 10:2244-7.

6. Mitch WE, Bailey JL, Wang X et al. Evaluation of signals activating ubiquitin-proteasone proteolysis in a model of muscle wasting. Am J Physiol 1999; 276:C1132-8.

7. Mehrotra R, Koople JD, Wolfson M. Metabolic acidosis in maintenance dialysis patients: clinical considerations. Kidney Int 2003; 64:S13-25.

8. Kalantar-Zadeh K, Mehrotra R, Fouque D, Kopple JD. Metabolic acidosis and malnutrition-inflamation complex syndrome in chronic renal failure. Semin Dial 2004; 17:455-65.

9. Chaveau P, Fouque D, Combe $\mathrm{C}$ et al. Acidosis and nutritional status in hemodialyzed patients. French Study Group for Nutrition in Dialysis. Semin Dial 2000; 13:241-6.

10. Graham KA, Hoenich NA, Goodship THJ. Pre-and interdialytic acid-base balance in hemodialysis patients. Int J Artif Organs 2001; 24:192-6.

11. Caso G, Garlick BA, Casélia GA, Sasvary D, Garlik PJ. Acute metabolic acidosis inhibits muscle protein synthesis in rats. Am J Physiol Endocrinol Metab 2004; 287:E90-6.

12. Bailey JL. Metabolic acidosis: An unrecognized cause of morbidity in the patient with chronic kidney disease. Kidney Int 2005; 68:S15-23.

13. Mitch WE, Hu Z, Woo Lee S, Du L. Strategies for suppressing muscle atrophy in chronic kidney disease: mechanisms activating distinct protealytic systems. J Renal Nutr 2005; 15:23-7. 
14. Mafra D, Burini RC. Efeitos da correção da acidose metabólica com bicarbonato de sódio sobre o catabolismo proteico na insuficiência renal crônica. Rev Nutr 2001; 14:53-9.

15. Pickering WP, Price SR, Bircher G, Marinovic AC, Mitch WE, Walls J. Metabolic Acidosis of Chronically Hemodialyzed Patients. Kidney Int 2002; 61:1286-92.

16. Movilli E, Bossini N, Viola BF et al. Evidence for an independent role of metabolic acidosis on nutritional status in haemodialysis patients. Nephrol Dial Transplant 1998; 13:674-8.

17. Williams AJ, Dittmer ID, McArley A, Clarke J. High bicarbonate dialysate in haemodialysis patients: effect on acidosis and nutritional status. Nephrol Dial Transplant 1997; 12:2633-7.

18. Szeto CC, Wong TY, Chow K, Leung C, Li PK. Oral sodium bicarbonate for the treatment of metabolic acidosis in peritoneal dialysis patients: a randomized placebo-control trial. J Am Soc Nephrol 2003; 14:2119. 26.

19. Lin SH, Lin YF, Chin HM, Wu CC. Must metabolic acidosis be associated with malnutrition in haemodialysed patients? Nephrol Dial Transplant 2002; 17:2006-10.

20. Williams AJ, Dittmer ID, McArley A, Clarke J. High bicarbonate dialysate in haemodialysis patients:effects on acidosis and nutritional status. Nephrol Dial Transplant 1997; 12:2633-7.

21. National Kidney Foundation: NKF-DOQI - I. Adult guidelines - A. Maintenance Dialysis - 2. Management of Acid-Base Status. New York: National Kidney Foundation, 2000.

22. National Kidney Foundation: NKF-DOQI - I. Clinical Practice Guidelines for Hemodialysis Adequacy - 1 . Measurement of Hemodialysis Adequacy. New York: National Kidney Foundation, 2000.

23. World Health Organization. Physical status: The use and interpretation of anthropometry. WHO Technical Report Series, 1995 , p. 854.

24. Frisancho AR. New norms of upper limb fat and muscle areas for assessment of nutritional status. Am J Clin Nutr 1981; 34:2540-5.

25. Kalantar-Zadeh K, Kleiner M, Dunne E, Lee GH, Luft FC. A modified quantitative subjective global assessment of nutrition for dialysis patients. Nephrol Dial Transplant 1999; 14:1732-8.

26. National Kidney Foundation: NKF-DOQI - I. Adult guidelines - A. Maintenance Dialysis - 1. Evaluation of Protein-Energy Nutritional Status. New York: National Kidney Foundation, 2000.

27. Martins C, Riella MC. Nutrição e Hemodiálise. In: Riella MC, Martins C. Nutrição e o Rim. Rio de Janeiro: Guanabara Koogan, 2001, pp. 114-31.

28. World Health Organization. Epi Info. Version 6.04. A word processing, database and statistic program for public health. [programa de computador]. Genebra, 1997.

29. Statistical Package for the Social Sciences for Windows Student version. Release 7.5. Marketing Department. Chicago, 1996.
30. Programa de Apoio a Nutrição. Versão 2.5. Centro de Informática em Saúde da Escola Paulista de Medicina - Universidade Federal de São Paulo - São Paulo/SP, 1993 (software).

31. Franco G. Tabela de composição química dos alimentos. São Paulo: Atheneu, 1997.

32. Pinheiro AVB, Lacerda EMA, Haimbenzecry E, Gomes MCS, Costa VM. Tabela para avaliação de consumo alimentar em medidas caseiras. Rio de Janeiro: [s.n.]; 1994. p. 74. (Produção Independente).

33. Locatelli F. Dose of dialysis, convection and haemodialysis patients outcome - what the HEMO study doesn't tell us: the European viewpoint. Nephrol Dial Transplant 2003; 18:1061-5.

34. Oettinger CW, Oliver JC. Normalization of uremic acidosis in hemodialysis patients with a high bicarbonate dialysate. J Am Soc Nephrol 1993; 3:1804-7.

35. Riella MC, Martins C. Avaliação e Monitorização do Estado Nutricional em Pacientes Renais. In: Riella MC, Martins C. Nutrição e o Rim. Rio de Janeiro: Guanabara Koogan, 2001, pp. 379-84.

36. Fouque D, Vennegoor M, Wee PT et al. EBPG Guideline on Nutrition. Nephrol Dial Transplant 2007; 22[Suppl. 2]:ii45-87.

37. Pifer TB, McCullough KP, Port FK et al. Mortality risk in hemodialysis patients and changes in nutritional indicators: DOPPS. Kidney Int 2002; 62:2238-45.

38. Blair D, Bigelow C, Sweet SJ. Nutritional effects of delivered bicarbonate dose in maintenance hemodialysis patients. J Renal Nutr 2003; 13:205-11.

39. Valenzuela RGV, Giffoni AG, Cuppari L, Canziani MEF. Estado nutricional de pacientes com insuficiência renal crônica em hemodiálise no Amazonas. Rev Assoc Med Bras 2003; 49:72-8.

40. Oe B, de Fijter CW, Oe PL, Stevens P, de Vries PM. Four-site skinfold anthropometry (FSA) versus body impedance analysis (BIA) in assessing nutritional status of patients on maintenance hemodialysis: which method is to be preferred in routine patient care? Clin Nephrol 1998; 49:180-5.

41. Goldstein DJ. Assessment of nutritional status in renal diseases. In: Mitch WE, Klahr S. Handbook of Nutrition and the Kidney, 3 ed. Philadelphia: Lippincott-Raven, 1998; p. 45

42. Seyffart G, Ensminger A, Scholz R. Increase of body mass during long-term bicarbonate hemodialysis. Kidney Int Suppl 1987; 22:S174-7.

43. Verove C, Maisonneuve N, El Azouzi A, Boldron A, Azer R. Effect of the correction of metabolic acidosis on nutritional status in elderly patients with chronic renal failure. J Renal Nutr 2002; 12:224-8.

44. Zheng ZH, Sederholm F, Anderstam B et al. Acute effects of peritoneal dialysis solutions on appetite in non-uremic rats. Kidney Int 2001; 60:2392-8.

45. Burrowes JD, Larive B, Chertow GM et al. Self-reported appetite, hospitalization and death in haemodialysis patients: findings from the hemodialysis (HEMO) Study. Neprhol Dial Transplant 2005; 20:2765-74. 